\title{
Cell Cycle Modulation of MCF-7 and MDA-MB-231 by a Sub- Fraction of Strobilanthes crispus and its Combination with Tamoxifen
}

\author{
Nik Soriani Yaacob ${ }^{1 *}$, Nik Nursyazni Nik Mohamed Kamal ${ }^{1}$, Kah Keng Wong ${ }^{2}$, \\ Mohd Nor Norazmi ${ }^{3}$
}

\begin{abstract}
Background: Cell cycle regulatory proteins are suitable targets for cancer therapeutic development since genetic alterations in many cancers also affect the functions of these molecules. Strobilanthes crispus (S. crispus) is traditionally known for its potential benefits in treating various ailments. We recently reported that an active sub-fraction of $S$. crispus leaves (SCS) caused caspase-dependent apoptosis of human breast cancer MCF-7 and MDA-MB-231 cells. Materials and Methods: Considering the ability of SCS to also promote the activity of the antiestrogen, tamoxifen, we further examined the effect of SCS in modulating cell cycle progression and related proteins in MCF-7 and MDA-MB-231 cells alone and in combination with tamoxifen. Expression of cell cyclerelated transcripts was analysed based on a previous microarray dataset. Results: SCS significantly caused G1 arrest of both types of cells, similar to tamoxifen and this was associated with modulation of cyclin D1, p21 and p53. In combination with tamoxifen, the anticancer effects involved downregulation of ER $\alpha$ protein in MCF-7 cells but appeared independent of an ER-mediated mechanism in MDA-MB-231 cells. Microarray data analysis confirmed the clinical relevance of the proteins studied. Conclusions: The current data suggest that SCS growth inhibitory effects are similar to that of the antiestrogen, tamoxifen, further supporting the previously demonstrated cytotoxic and apoptotic actions of both agents.
\end{abstract}

Keywords: Strobilanthes crispus - tamoxifen - cell cycle - estrogen receptor - MCF-7 - MDA-MB-231

Asian Pac J Cancer Prev, 16 (18), 8135-8140

\section{Introduction}

Many cancer patients seek traditional therapeutic medicines with the hope of enhancing their cancer therapy while minimizing side effects. Others may seek such treatments due to the limited access to conventional pharmacological treatment. As such, interest in the therapeutic use of natural products has grown with many researchers now focussing on medicinal plants as a source of anticancer remedies or as adjuvants to enhance the efficacy of conventional chemotherapeutic drugs.

Cell proliferation is tightly regulated by a number of different molecules that control the balance between growth promoting and growth inhibiting signals. Dysregulation of normal cell division processes results in uncontrolled cell proliferation that may lead to cancer development. Cell cycle checkpoints monitor the cellular environment and control cell cycle transition. Cyclins and cyclin-dependent kinases (CDKs) accelerate cell cycle progression, while CDK inhibitors such as p21 and p27, and tumor suppressor proteins, p53 and RB, halt its progression (Garrett, 2001). Abnormalities of cell cycle regulators in cancers are commonly reported, making cell cycle regulators important potential targets for chemopreventive treatments (Stewart et al., 2003). In the current study, a bioactive subfraction of Strobilanthes crispus (SCS) that was previously shown to be potent inhibitors of breast and prostate cancer cell growth (Yaacob et al., 2010) was investigated for its ability to modulate cell cycle progression in MCF-7 and MDAMB-231 breast cancer cells. SCS was recently found to synergise with tamoxifen to induce apoptosis of these cancer cell lines (Yaacob et al., 2014). Hence, the effect of SCS on tamoxifen-induced cell cycle-related activities was explored. Herein we showed that SCS alone and in combination with tamoxifen induced cell cycle arrest and modulated specific cell cycle regulatory proteins that are reported to be abnormally expressed in human breast adenocarcinoma.

\section{Materials and Methods}

\section{Plant material}

The leaves of S. crispus (L.) Blume were purchased

${ }^{1}$ Department of Chemical Pathology, ${ }^{2}$ Department of Immunology, School of Medical Sciences, ${ }^{3}$ School of Health Sciences, Universiti Sains Malaysia Health Campus, Kubang Kerian, Kelantan, Malaysia*For correspondence: niksoriani@usm.my, sorianiyaacob@ gmail.com 
Nik Soriani Yaacob et al

from Agrodynamic Resources (Malaysia) that cultivated the plant in Tasek Gelugor, Pulau Pinang. A voucher specimen was deposited at the university herbarium (no. 11046) following plant authentication. SCS was prepared by the Centre for Drug Research, Universiti Sains Malaysia, as previously described (Yaacob et al., 2010) and kept at $-20^{\circ} \mathrm{C}$ as a $10 \mathrm{mg} / \mathrm{ml}$ stock in DMSO (Sigma-Aldrich, USA).

\section{Antibodies}

Rabbit anti-human $\beta$-actin polyclonal $\mathrm{IgG}$, rabbit anti-human cyclin A polyclonal IgG and goat polyclonal secondary antibody to rabbit IgG-H\&L (HRP) were purchased from Abcam (UK). Rabbit anti-human cyclin B1 polyclonal IgG, rabbit anti-human cyclin D1 polyclonal IgG, rabbit anti-human cyclin E2 polyclonal IgG, rabbit anti-human p21 polyclonal IgG and rabbit anti-human $\mathrm{p} 53$ polyclonal IgG antibodies were purchased from Cell Signaling Technology (USA).

\section{Cell culture and treatment}

MCF-7 and MDA-MB-231 breast cancer cell lines (ATCC, USA) were maintained in Rosselle's Park Memorial Institute medium (RPMI-1640) and Dulbeccomodified Eagle's medium (DMEM), respectively, with $10 \%$ fetal bovine serum (Hyclone, USA) and 50 units $/ \mathrm{ml}$ penicillin/streptomycin (GIBCO BRL, UK) at $37^{\circ} \mathrm{C}$ in an atmosphere of $5 \% \mathrm{CO}_{2}$. Tamoxifen (Sigma-Aldrich, USA) was prepared as a $10 \mathrm{mM}$ stock in ethanol. Cells were seeded up to $70 \%$ confluence and then treated with SCS at 8.5 or $10.0 \mu \mathrm{g} / \mathrm{ml}$ as previously reported (Yaacob et al., 2010), $5 \mu \mathrm{M}$ tamoxifen or their combination for $24 \mathrm{~h}$ (cell cycle analyses by flow cytometry) and up to $48 \mathrm{~h}$ for Western blot analyses.

\section{Cell cycle analysis}

Prior to treatment for cell cycle analysis, the cells were serum starved (using serum-free medium) for 24 $h$ to synchronize them in $G_{0}$ phase of the cell cycle. Cell cycle distribution was assayed using Cycle TEST ${ }^{\mathrm{TM}}$ PLUS DNA Reagent Kit (Becton Dickinson, USA) according to the manufacturer's guideline and analyzed by the Becton Dickinson FACScan apparatus. In brief, both floating and adherent cells were harvested, followed by centrifugation and washing with ice-cold PBS. Cells $\left(1 \times 10^{6}\right)$ were incubated with propidium iodide in the presence of RNase A for $30 \mathrm{~min}$ at $4^{\circ} \mathrm{C}$ in the dark. Data from at least 15,000 cells per sample were acquired and analyzed using the ModFit LTTM software (USA).

\section{Protein extraction and Western blotting}

Cell lysates were harvested by centrifugation at 12,000 rpm for 2 min following lysis in lysis buffer $(50 \mathrm{mM}$ of Tris- $\mathrm{HCl}, 150 \mathrm{mM}$ of $\mathrm{NaCl}, 0.2 \%$ SDS, $1 \mathrm{mM}$ PMSF, 2 $\mu \mathrm{g} / \mathrm{ml}$ of leupeptin, $2 \mu \mathrm{g} / \mathrm{ml}$ of aprotinin and $1 \mathrm{mM}$ of $\mathrm{Na}_{3} \mathrm{VO}_{4}$ ). The protein concentrations were determined spectrophotometrically (Thermoscientific, USA) and samples of 50-100 $\mu \mathrm{g}$ were resolved using $10 \%$ SDSpolyacrylamide gel followed by semi-dry transfer onto the PVDF membrane. The proteins were immunoblotted for cyclins A, B, D1 and E, p21, p53 and $\beta$-actin overnight at $4^{\circ} \mathrm{C}$ and subsequently reacted with horseradish peroxidaseconjugated secondary antibody. Antibody-bound proteins were detected by chemiluminescence using ECL ${ }^{\mathrm{TM}}$ Prime Western Blotting Detection reagent (GE Healthcare, UK) according to the manufacturer's protocols and visualised using the Fluor chem FC2 image analyser (DKSH, US). The band density for each treatment compared to control was analyzed using ImageJ 1.46 software (http://imagej. nih.gov/ij/) and normalized to the $\beta$-actin band density.

\section{Microarray data-mining}

A microarray dataset (GEO accession number: GSE3744; Richardson et al., 2006) containing transcript values of CCNE1 (probeset ID: 213523_at), CCNA2 (203418_at), CCNB1 (228729_at), CCND1 (208711_at), ESR1 (215551_at), ESR2 (1569554_at), CDKN1A (202284_s_at) or TP53 (201746_at) in ductal breast carcinomas $(n=40)$ and non-malignant breast tissues $(n=7)$ of human origin were obtained from Oncomine (https:// www.oncomine.org) and analyzed.

\section{Statistical analysis}

Statistical analyses for DNA content and protein expression profiles were performed using IBM SPSS Statistics 20 (Chicago, IL, USA) and significance levels were evaluated using ANOVA with Tukey's multiple comparison test and $\mathrm{p}<0.05$ was considered as significantly different from control. Bioinformatics analysis comparing the transcript values between malignant and non-malignant breast tissues was carried out using Mann-Whitney test.

\section{Results}

Induction of cell cycle arrest in MCF-7 and MDA-MB-231 cells

Following our recent findings that SCS synergises with tamoxifen to induce death of breast cancer cells (Yaacob et al., 2014), we hypothesized that this involved inihibition of cell cycle progression. The DNA content of cells treated with SCS, tamoxifen and their combination was first measured by flow cytometry. SCS significantly increased $(\mathrm{p}<0.05)$ the percentage of MCF-7 cells in G1 (73.4\%) and $\mathrm{G} 2 / \mathrm{M}$ phases $(9.4 \%$ ) with reduced percentage of cells in S phase (17.2\%) compared to untreated controls (58.7, 5.4 and $35.9 \%$, respectively; Figure 1A). Similarly, $5 \mu \mathrm{M}$ tamoxifen either alone or in combination with SCS, caused accumulation of cells in G1 phase (72.8 and $70.6 \%$, respectively) with a corresponding decrease in the percentage of cells in the S phase (22.3 and $23.1 \%$, respectively) compared to untreated controls. These results suggest that SCS, tamoxifen and their combination exerted their growth suppressive activity in MCF-7 cells by arresting cell cycle progression at $\mathrm{G} 1$ phase. The combined SCS and tamoxifen however, caused similar levels of cell cycle arrest compared to either agent alone.

As with MCF-7 cells, SCS treatment resulted in significant accumulation of MDA-MB-231 cells in G1 phase $(74.0 \%)$ while reducing the percentage of cells in $\mathrm{S}$ phase $(19.4 \% ; \mathrm{p}<0.05)$ compared to control cultures $(51.3$ and $34.8 \%$, respectively) (Figure 1C). However, there 
was no significant change in the cell population in $\mathrm{G} 2 / \mathrm{M}$ phase. Treatment with tamoxifen or the combination of tamoxifen and SCS also resulted in a significant increase in the population of MDA-MB-231 cells in G1 phase (63.5 and $70.2 \%$, respectively) compared to controls. A corresponding decrease in the percentage of cells in the $\mathrm{S}$ phase was also evident following tamoxifen $(26.4 \%)$ and SCS+tamoxifen (18.9\%). The results thus far indicate that the mechanism of growth inhibitory effect of SCS is similar to that of tamoxifen in both types of breast cancer cells.

Effects on cell cycle regulatory proteins in $M C F-7$ and MDA-MB-231 cells

To further explore the mechanism of cell growth arrest by SCS and tamoxifen, we determined the expression of cyclins, p21 CDK inhibitor and p53 tumor suppressor protein, in $24 \mathrm{~h}$ - and $48 \mathrm{~h}$-treated and untreated cells. We observed a three-fold increase in the expression of cyclin D1 after $24 \mathrm{~h}$ treatment of MCF-7 cells with SCS which was accompanied with increased p21 and p53 expression. This was then followed by a decrease in cyclin D1 expression at $48 \mathrm{~h}$, similar to the control level (Figure 2A). Similarly, tamoxifen alone or in combination with SCS markedly decreased cyclin D1 expression while increased p21 and p53 expression at $48 \mathrm{~h}$ post-treatment compared to the control. The expression of cyclin $\mathrm{E}$ was found to be reduced by SCS and the combination treatment while that of cyclins A and B remained similar to control level.

In contrast to the observations in MCF-7 cells, SCS decreased cyclin D1 protein expression in MDA-MB-231 cells at both $24 \mathrm{~h}$ and $48 \mathrm{~h}$ (Figure 2B). Although tamoxifen alone had no effects on the expression of cyclin D1, its combination with SCS caused a reduction in this expression. The expression of cyclins E, A and B in these cells was not significantly modulated by SCS or tamoxifen alone. However, reduced expression of cyclin $\mathrm{E}$ was observed following the combination treatment while
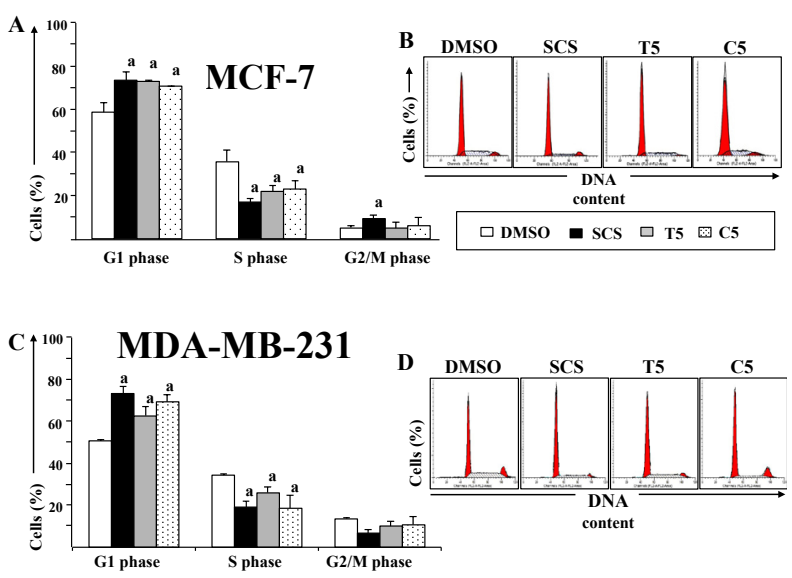

Figure 1. Effect of SCS, Tamoxifen and SCS+Tamoxifen on the Cell Cycle of MCF-7 and MDA-MB-231 Cells. Percentage of cells in $\mathrm{G} 1, \mathrm{~S}$ and G2/M phases were analysed by flow cytometry after $24 \mathrm{~h}$ treatment with 8.5 or $10.0 \mu \mathrm{g} / \mathrm{ml} \mathrm{SCS}$, $5 \mu \mathrm{M}$ tamoxifen (T5) or their combination (C5). The results are the means of three independent experiments $(A, C)$. Letter ' $a$ ' indicates significant values at $\mathrm{p}<0.05$, compared to control cells (DMSO-treated). Representative histograms showing cyclin B expression was slightly increased at $48 \mathrm{~h}$. The expression of $\mathrm{p} 53$ protein was not significantly modulated by all treatments.

Effects on the ER protein expression in both MCF-7 and MDA-MB-231 cells

The ER $\alpha$-positive MCF-7 cells depend on estrogens acting on ER $\alpha$ for proliferation (Helguero et al., 2005). However, our results showed that SCS or tamoxifen did not interfere with the constitutive expression of ER $\alpha$ nor ER $\beta$ protein in the hormone-dependent MCF-7 cells (Figure 3 ) indicating that cell growth inhibition of these cells by the individual agents does not involve ER regulation. Interestingly, treatment with the combination of SCS and tamoxifen for $48 \mathrm{~h}$ led to a four-fold reduction

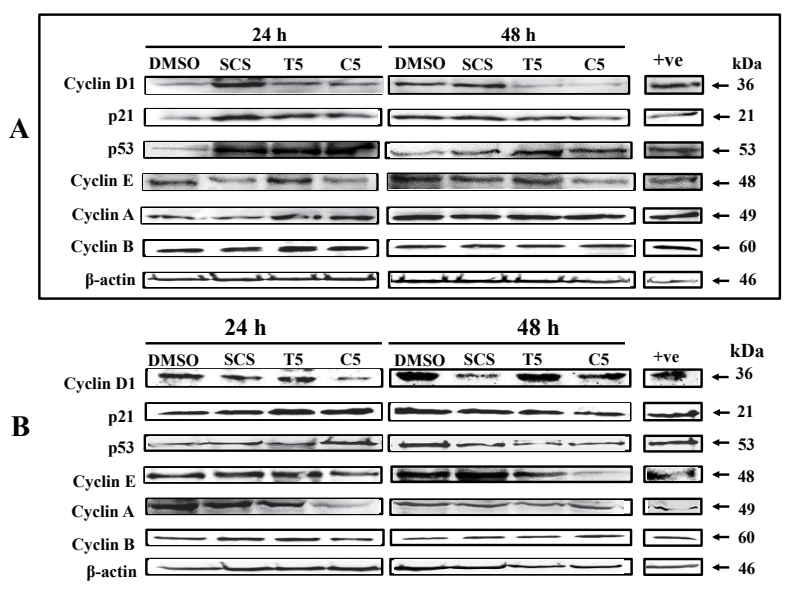

Figure 2. Cell Cycle-related Proteins in MCF-7 and MDA-MB-231 Cells Treated with SCS, Tamoxifen and SCS+Tamoxifen. MCF-7 (A) and MDA-MB-231 (B) cells treated with SCS (8.5 and $10.0 \mu \mathrm{g} / \mathrm{ml}$, respectively), tamoxifen (T5; $5 \mu \mathrm{M})$ or SCS+tamoxien (C5) for $24 \mathrm{~h}$ and 48 $\mathrm{h}$ were harvested for preparation of total protein and Western blotting. DMSO $(0.1 \% \mathrm{v} / \mathrm{v})$ was used for the control cultures. The band density for each treatment compared to control was analyzed using ImageJ 1.46 software (http://imagej.nih.gov/ij/) and normalized to the $\beta$-actin band density
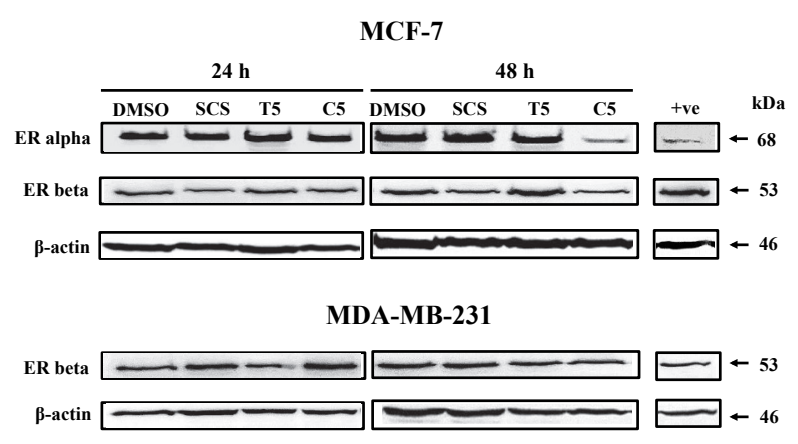

Figure 3. ER $\alpha$ and ERß Expression in MCF-7 and MDA-MB-231 Cells Treated with SCS, Tamoxifen and SCS+Tamoxifen. MCF-7 and MDA-MB-231 cells treated with SCS ( 8.5 and $10.0 \mu \mathrm{g} / \mathrm{ml}$, respectively), tamoxifen (T5; 5 $\mu \mathrm{M}$ ) or SCS+tamoxien (C5) for $24 \mathrm{~h}$ and $48 \mathrm{~h}$ were harvested for preparation of total protein and Western blotting. DMSO $(0.1 \% \mathrm{v} / \mathrm{v})$ was used for the control cultures. The band density for each treatment compared to control was analyzed using ImageJ 1.46 software (http://imagej.nih.gov/ij/) and normalized to the $\beta$-actin band density 


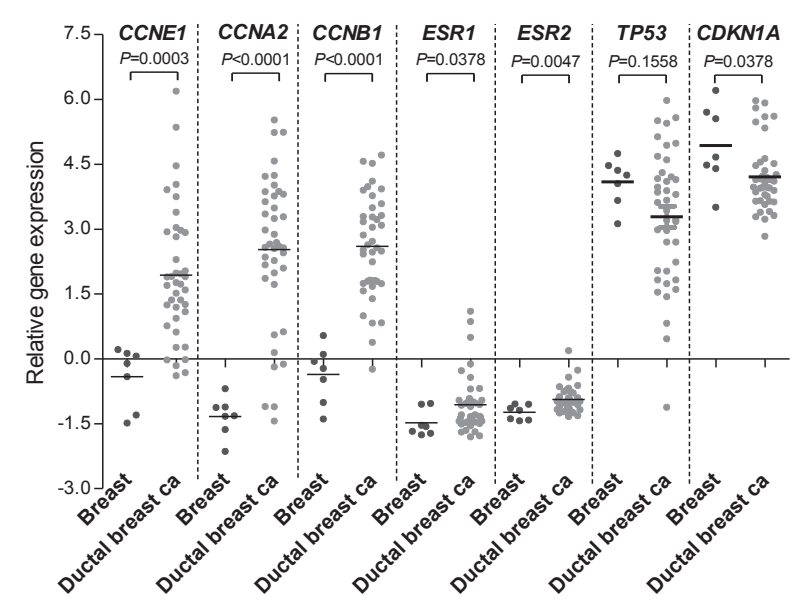

Figure 4. Expression of Cell Cycle-related Genes in Breast Carcinomas And Nonmalignant Tissues. The transcript levels of CCNE1, CCNA2, CCNB1, ESR1, ESR2, TP53 and CDKN1A in human ductal breast carcinomas $(n=40)$ and non-malignant breast tissues $(n=7)$ were analysed from a microarray dataset (GEO accession number: GSE3744; Richardson et al., 2006)

in ER $\alpha$ expression in MCF-7 cells. On the other hand, MDA-MB-231 cells are deficient of ER $\alpha$ but expresses ER $\beta$. Nevertheless, ER $\beta$ expression in these cells was also not affected by any of the treatments.

Expression of cell cycle-related genes in breast carcinomas and non-malignant tissues

Analysis of the transcript levels in a published microarray study (Richardson et al., 2006) demonstrated that cyclins $\mathrm{E}(C C N E 1), \mathrm{A}(C C N A 2)$ and $\mathrm{B}(C C N B 1)$ genes were significantly more highly expressed in ductal breast carcinomas than in non-malignant breast tissues of human origin (Figure 4). The gene coding for cyclin $\mathrm{D}(C C N D 1)$ also showed a trend towards increased expression in breast carcinomas (when tested with significant unpaired t-test). On the other hand, although there was significant downregulation of p21 (CDKN1A) in breast cancer, p53 transcription level does not significantly differ from normal subjects. ESR 1 and $E S R 2$ genes coding for $E R \alpha$ and $E R \beta$, respectively, were also significantly upregulated in the breast cancer tissues. These observations confirm the clinical relevance of these proteins in breast cancer, in line with the analysis of SCS effects on these proteins described in this study.

\section{Discussion}

Our previous results suggested that SCS could act as an adjuvant that enhanced the apoptotic activities of tamoxifen in breast cancer cells (Yaacob et al., 2014). Apoptosis is commonly associated with cell cycle arrest as both mechanisms involve common genes or molecules (Choi et al., 2001; Alenzi, 2004). For example, the tumor suppressor protein, p53, is known to affect both cellular growth arrest and apoptosis, primarily through transactivation of molecules such as p21, Gadd45, Mdm2, cyclin D1, Bax, Bcl-xL and FasL (Alenzi, 2004; Pfaum et al., 2014). Herein we show that SCS significantly induced cell cycle arrest of both ER $\alpha$-positive MCF-7 and ER $\alpha$ - negative MDA-MB-231 cells at G1 phase, similar to the action of the commonly used antiestrogen, tamoxifen. A number of anti-cancer agents derived from plants are also reported to inhibit cancer cell growth by blocking cell cycle progression. For example, quercetin (a polyphenol) arrested breast cancer cells at G1 or G2/M phase (Choi et al., 2001), curcumin (derived from turmeric of Curcuma longa) caused G2/M arrest in bladder cancer cells (Park et al., 2006) and gambogic acid (isolated from gamboge resin of Garcinia hanburryi) inhibited growth of gastric carcinoma cells by inducing G2/M arrest (Yu et al., 2006).

Microarray data mining of a gene expression dataset (Richardson et al., 2006) presented herein indicates that genes coding for cyclins were abnormally expressed in breast cancer suggesting that dysregulation of the cell cycle associated with abnormal expression of cell cycle-related proteins is an important mechanism in breast cancer pathogenesis. Cyclin D1 for example is commonly overexpressed in approximately $50 \%$ of breast cancer cases (Fu et al., 2004). Deregulation of cyclin D1 expression alters cell division cycle of normal cells to be less dependent on growth factors and therefore accelerates passage through the G1 phase (Alao, 2007). Herein, we determined the expression of cyclin D1, E, $\mathrm{A}$ and $\mathrm{B}$ proteins and found that cyclin D1 expression was modulated by SCS and tamoxifen in MCF-7 cells. Previous studies demonstrated that reduction in cyclin D1 protein expression is an early and critical event in antiestrogen action (Doisneau-Sixou et al., 2003; Butt et al., 2005). This is consistent with our findings of twofold reduction in cyclin D1 protein level following 48 $\mathrm{h}$ tamoxifen treatment. Cyclin D1 complexes with and regulates the activity of CDK4 or CDK6 that mediates phosphorylation of the retinoblastoma gene product, $\mathrm{pRb}$, required for G1/S transition (Lange and Yee, 2011).

Cyclin D1 expression can mimic the action of estrogen on cell cycle progression and re-initiate cell proliferation in antiestrogen-arrested cells (Butt et al., 2005). Therefore, overexpression of cyclin D1 may cause sustained ER signaling and development of endocrine resistance in breast cancer cells (Hui et al., 2002; Butt et al., 2005). In our study, cyclin D1 expression in MCF-7 cells was initially increased by SCS at $24 \mathrm{~h}$ but then declined to control level after $48 \mathrm{~h}$ treatment. This could suggest an initial response by the cancer cells to develop resistance against SCS treament. This effect was however abrogated with longer exposure to SCS, or combined treatment of SCS and tamoxifen which resulted in a 2.5 -fold reduction in cyclin D1 expression, similar to the effect of the antiestrogen alone. In addition, the increased cyclin D1 expression was accompanied with elevated p21 and p53 expression. The effects of cyclin D1 overexpression was proposed to be neutralized by a feedback mechanism between the positive (cyclin D1) and negative (p21) regulators of the cell cycle, which ultimately resulted in proliferation arrest (de Jong et al., 1999). Thus, p21 elevation in the current study may serve as a feedback response to the apparent increase in cyclin $\mathrm{D} 1$ protein level induced by SCS in MCF-7 cells. The increased p53 expression is in agreement with previous reports that p53 increases the transcription of several genes, including 
p21, followed by arrest of cells at G1 phase (Ichikawa et al., 2008; Jedinak et al., 2008). Similarly, Chong and colleagues (Chong et al., 2012) demonstrated an increase in p53 protein level following $S$. crispus ethanol extract treatment of MCF-7 cells. It is therefore postulated that induction of $\mathrm{p} 21$ and $\mathrm{p} 53$ expression contributes to the G1 phase arrest and apoptotic effects of SCS in MCF-7 cells.

Interestingly, the initial upregulation of cyclin D1 protein was not observed in the ER $\alpha$-negative MDAMB-231 cells in which a 2-fold and 5-fold reduction were noted with SCS alone or in combination with tamoxifen, respectively. MDA-MB-231 cells contain mutant p53 and no concomitant increase in $\mathrm{p} 53$ or p 21 expression was observed, suggesting that the inhibition of MDA-MB-231 cell growth arrest by SCS may involve a mechanism different from that of MCF-7 cells. Overexpression of mutated p53 would be undesirable as it could provide the cells with growth advantage. The current finding is also consistent with the reported ability of phenetyl isoisothiocyanate (derived from watercress plant) and conjugated linoleic acids (derived from linoleic acid) to selectively suppress mutant $\mathrm{p} 53$, which was followed by apoptotic cell death in MDA-MB-231 cells (Majumder et al., 2002; Wang et al., 2011).

Cyclins A and B that are associated with S phase and G2/M transition, respectively, were not affected by SCS treatment indicating insignificant role in SCS mechanism of action in both MCF-7 and MDA-MB-231 cells. Cyclin $\mathrm{B}$ expression was however, increased with the combined SCS and tamoxifen at $48 \mathrm{~h}$. As activation of Cdc2/cyclin $\mathrm{B}$ complex is required for cell progression from $\mathrm{G} 2$ to $M$ phase and binding of p21 to this complex inhibits its activity (Garrett, 2001), the increased expression of cyclin $B$ could be a consequence of the lack of $\mathrm{p} 21$ upregulation in MDA-MB-231 cells which unlike MCF-7 cells, are p53defective. On the other hand, the reduced expression of cyclin $\mathrm{E}$ by SCS alone or in combination with tamoxifen may cause disruption of the formation of active CDK2/ cyclin $\mathrm{E}$ complex required for phosphorylation of $\mathrm{pRb}$ and activation of E2F transcription factor, which are crucial for $\mathrm{G} 1 / \mathrm{S}$ transition (Garrett, 2001). This provides support for the G1 phase arrest induced by SCS above.

The effects of estrogen are mediated via two estrogen receptors, $\mathrm{ER} \alpha$ that mediates proliferation and $\mathrm{ER} \beta$ that exerts proapoptotic effects in cancer cells, such as with breast and prostate cancer cell lines (Helguero et al., 2005). ER $\beta$ is co-expressed with $E R \alpha$ in many human breast cancers (Kurebayashi et al., 2000) but is expressed at low levels compared to normal tissues (Freund et al., 2003). ER $\alpha$ and ER $\beta$ have similar DNA-binding domains but differ in the ligand-binding domains especially in the $\mathrm{N}$-terminal transactivation domain (Katzenellenbogen et al., 2000). Studies suggest that ER $\beta$ negatively regulates some of ER $\alpha$ functions (Lindberg et al., 2003; Paruthiyil et al., 2004). The opposing action of ER $\beta$ on cell proliferation potentially occurs via repression of cyclin D1 and cyclin A expression and upregulation of growth inhibitory molecules like p21 and p27 (Paruthiyil et al., 2004). SCS alone did not alter the expression of $\mathrm{ER} \alpha$ in MCF-7 cells but caused a four-fold decrease in ER $\alpha$ protein expression when combined with tamoxifen.
Although upregulation of p21 was observed in MCF-7 cells, no significant changes in ER $\beta$ expression were observed following all treatments of MCF-7 cells as well as the ER $\alpha$-negative MDA-MB-231 cells. We could therefore conclude that SCS anticancer activities involved downregulation of ER $\alpha$ protein in MCF-7 cells but are independent of ER-mediated mechanism in MDA-MB-231 cells. Steigerova et al. (2010) showed that brassinosteroids (plant hormones) caused cell cycle arrest and apoptosis of MCF-7 and MDA-MB-435 cancer cells via modulation of $E R \alpha$ but not $E R \beta$ expression, which is in agreement with our current findings.

In conclusions, our data suggest that the growth inhibitory effects of SCS is similar to that of the antiestrogen, tamoxifen which provides further support to their synergistic cytotoxic and apoptotic action reported earlier (Yaacob et al., 2014). Its growth inhibitory action either alone or in combination with tamoxifen, is partly attributed to suppression of $E R \alpha$ and induction of $\mathrm{p} 21$ and p53 leading to G1 phase arrest of MCF-7 cells. In the ER $\alpha$-negative MDA-MB-231 cells, the mechanism involves downregulation of cyclin D1 and upregulation of p21 as well as suppression of the mutated p53 protein. The specific components of SCS that possess the antiestrogenic and anticancer effects have yet to be determined.

\section{Acknowledgements}

The research was funded by the USM Research University Grant (1001.PPSP.853002), and the Malaysian Ministry of Science, Technology and Innovation/IPharm Research Initiative Grant (304.PPSP.6150098). NNNMK received the USM Fellowship for her postgraduate study.

\section{References}

Alao JP (2004). The regulation of cyclin D1 degradation, roles in cancer development and the potential for therapeutic invention. Mol Cancer, 6, 24.

Alenzi FQB (2004). Links between apoptosis, proliferation and the cell cycle. Br J Biomed Sci, 61, 99-102.

Butt AJ, McNeil CM, Musgrove EA, Sutherland RL (2005). Downstream targets of growth factor and oestrogen signalling and endocrine resistance, the potential roles of c-Myc, cyclin D1 and cyclin E. Endocr-Relat Cancer, 12, 547-59.

Choi JA, Kim JY, Lee JY, et al (2001). Induction of cell cycle arrest and apoptosis in human breast cancer cells by quercetin. Int J Oncol, 19, 837-44.

Chong HZ, Rahmat A, Yeap SK, et al (2012). In vitro cytotoxicity of Strobilanthes crispus ethanol extract on hormone dependent human breast adenocarcinoma MCF-7 cells. BMC Complement Altern Med, 12, 35.

de Jong JS, van Diest PJ, Michalides RJAM, Baak JPA (1999). Concerted overexpression of the genes encoding p21 and cyclin D1 is associated with growth inhibition and differentiation in various carcinomas. Mol Pathol, 52, 78-83.

Doisneau-Sixou SF, Sergio CM, Carroll JS, et al (2003). Estrogen and antiestrogen regulation of cell cycle progression in breast cancer cells. Endocr-Relat Cancer, 10, 179-86.

Freund A, Chauveau C, Brouillet J-P, et al (2003). IL-8 expression and its possible relationship with estrogen-receptor-negative status of breast cancer cells. Oncogene, 22, 256-65. 


\section{Nik Soriani Yaacob et al}

Fu M, Wang C, Li Z, Sakamaki T, Pestell RG (2004). Cyclin D1, normal and abnormal functions. Endocrinol, 145, 5439-47.

Garrett MD (2001). Cell cycle control and cancer. Curr Sci, $81,515-22$.

Helguero LA, Faulds MH, Gustadsson JA, Haldosen LA (2005). Estrogen receptor alpha $(E R \alpha)$ and beta $(E R \beta)$ differentially regulate proliferation and apoptosis of the normal murine mammary epithelial cell line HC11. Oncogene, 24, 6605-16.

Hui R, Finney GL, Carroll JS, et al (2002). Constitutive overexpression of cyclin D1 but not cyclin E confers acute resistance to antiestrogen in T-47D breast cancer cells. Cancer Res, 62, 6916-23.

Ichikawa A, Ando J, Suda K (2008). G1 arrest and expression of cyclin-dependent kinase inhibitors in tamoxifen-treated MCF-7 human breast cancer cells. Human Cell, 21, 28-37.

Jedinak A, Sliva D (2008). Pleurotus ostreatus inhibits proliferation of human breast and colon cancer cells through p53-dependent as well as p53-independent pathway. Int $J$ Oncol, 33, 1307-13.

Katzenellenbogen BS, Montano MM, Ediger TR, et al (2000). Estrogen receptors, selective ligands, partners, and distinctive pharmacology. Recent Prog Horm Res, 55, 163-93.

Kurebayashi J, Otsuki T, Kunisue H, et al (2000). Expression levels of estrogen receptor- $\alpha$, estrogen receptor- $\beta$, coactivators, and corepressors in breast cancer. Clin Cancer Res, 6, 512-8.

Lange CA, Yee D (2011). Killing the second messenger, targeting loss of cell cycle control in endocrine-resistant breast cancer. Endocr-Relat Cancer, 18, 19-24.

Lindberg MK, Moverare S, Skrtic S, et al (2003). Estrogen receptor (ER)- $\beta$ reduces ER $\alpha$-regulated gene transcription, supporting a "ying yang" relationship between ER $\alpha$ and ER $\beta$ in mice. Mol Endocrinol, 17, 203-8.

Majumder B, Wahle KWJ, Moir S, et al (2002). Conjugated linoleic acids (CLAs) regulate the expression of key apoptotic genes in human breast cancer cells. FASEB $J$, 16, 1447-9.

Park C, Kim GY, Kim GD, et al (2006). Induction of G2/M arrest and inhibition of cyclooxygenase- 2 activity by curcumin in human bladder cancer T24 cells. Oncol Rep, 15, 1225-31.

Paruthiyil S, Parmar H, Kerekatte V, et al (2004). Estrogen receptor $\beta$ inhibits human breast cancer cell proliferation and tumor formation by causing a G2 cell cycle arrest. Cancer Res, 64, 423-8.

Pflaum J, Schlosser S, Muller M (2014). p53 family and cellular stress responses in cancer. Front Oncol, 4, 285.

Steigerova J, Oklest'kova J, Levkova M, et al (2010). Brassinosteroids cause cell cycle arrest and apoptosis of human breast cancer cells. Chem Biol Interact, 188, 487-96.

Richardson AL, Wang ZC, De Nicolo A, et al (2006). X chromosomal abnormalities in basal-like human breast cancer; Cancer Cell, 9, 121-32.

Stewart ZA, Westfall MD, Pietenpol JA (2003). Cell-cycle dysregulation and anticancer therapy. Trends Pharmacol Sci, 24, 139-45.

Wang X, Di Pasqua AJ, Govind S, et al (2011). Selective depletion of mutant p53 by cancer chemopreventive isothiocyanates and their structure-activity relationships. $J$ Med Chem, 54, 809-16.

Yaacob NS, Hamzah N, Nik Mohamed Kamal NN, et al (2010). Anticancer activity of a sub-fraction of dichloromethane extract of Strobilanthes crispus on human breast and prostate cancer cells in vitro. BMC Complement Altern Med, 10, 42.

Yaacob NS, Nik Mohamed Kamal NN, Norazmi MN (2014). Synergistic anticancer effects of a bioactive subfraction of Strobilanthes crispus and tamoxifen on MCF-7 and MDAMB-231 human breast cancer cell lines. BMC Complement
Altern Med, 14, 252.

Yu J, Guo QL, You QD, et al (2006). Gambogic acid-induced G2/M phase cell-cycle arrest via disturbing CDK7-mediated phosphorylation of $\mathrm{CDC} 2 / \mathrm{p} 34$ in human gastric carcinoma BGC-823 cells. Carcinogenesis, 28, 632-8. 